\title{
Reflets
}

Revue ontaroise d'intervention sociale et communautaire

\section{La loi 68 : loi en l'honneur de Brian Smith}

\section{Julie Gagnon}

Volume 7, numéro 1, printemps 2001

Santé mentale et les défis de l’an 2001

URI : https://id.erudit.org/iderudit/026335ar

DOI : https://doi.org/10.7202/026335ar

Aller au sommaire du numéro

Éditeur(s)

Reflets : Revue ontaroise d'intervention sociale et communautaire

ISSN

1203-4576 (imprimé)

1712-8498 (numérique)

Découvrir la revue

Citer cet article

Gagnon, J. (2001). La loi 68 : loi en l'honneur de Brian Smith. Reflets, 7(1), 26-47. https://doi.org/10.7202/026335ar

\section{Résumé de l'article}

$\mathrm{Au}$ cours de l'hiver 2000, le gouvernement ontarien annonçait la venue prochaine desordonnances de traitement communautaire obligatoire pour les psychiatrisés. Méthode d'intervention controversée, le traitement coercitif soulève plusieurs enjeux, pratiques et éthiques, dont plusieurs sont liés aux droits et libertés des psychiatrisés. Dans ce travail, nous étudions la question du traitement communautaire obligatoire. Nous décrivons son concept et son contexte. Nous identifions les partisans et les opposants au traitement coercitif, tout comme leurs arguments à l'appui. Nous soulevons les thèmes et les enjeux qui ressortent de ce débat. Nous suggérons d'ailleurs des interventions alternatives au traitement communautaire obligatoire.
Tous droits réservés (C) Reflets : Revue ontaroise d'intervention sociale et communautaire, 2001
Ce document est protégé par la loi sur le droit d'auteur. L'utilisation des services d'Érudit (y compris la reproduction) est assujettie à sa politique d'utilisation que vous pouvez consulter en ligne.

https://apropos.erudit.org/fr/usagers/politique-dutilisation/ 


\section{La loi 68 : loi en l'honneur de Brian Smith}

Au cours de l'hiver 2000, le gouvernement ontarien annonçait la venue prochaine des ordonnances de traitement communautaire obligatoire pour les psychiatrisés. Méthode d'intervention controversée, le traitement coercitif soulève plusieurs enjeux, pratiques et éthiques, dont plusieurs sont liés aux droits et libertés des psychiatrisés.

Dans ce travail, nous étudions la question du traitement communautaire obligatoire. Nous décrivons son concept et son contexte. Nous identifions les partisans et les opposants au traitement coercitif, tout comme leurs arguments à l'appui. Nous soulevons les thèmes et les enjeux qui ressortent de ce débat. Nous suggérons d'ailleurs des interventions alternatives au traitement communautaire obligatoire.

Julie Gagnon, Maîtrise en service social

Intervenante en santé mentale, Ottawa Salus Corporation

\section{Introduction}

Ontario to Introduce Stronger Mental Health Law this Spring to Help Patients and Protect Communities (OMH 2000a :1). Tel était le titre que nous pouvions lire dans le relevé de presse du gouvernement ontarien et dans certains journaux de la région, au mois de mars 2000. Élizabeth Witmer, ministre de la Santé et des soins de longue durée (Ontario), annonçait la venue prochaine des ordonnances de traitement communautaire (OTC) pour les psychiatrisés. La loi 68, loi en l'honneur de Brian Smith (journaliste tué par un homme atteint de schizophrénie), est proclamée le $1^{\text {er }}$ décembre 
"Le traitement obligatoire soulève plusieurs enjeux, surtout par rapport aux droits et aux libertés des personnes. Il semble être une autre source de discrimination envers les psychiatrisés. "
2000 (OMH 2000d : 1). Cette loi controversée permet, entre autres, d'imposer un traitement communautaire obligatoire aux personnes qui souffrent de problèmes de santé mentale $(\mathrm{OMH}$ 2000d : 1).

Le traitement obligatoire soulève plusieurs enjeux, surtout par rapport aux droits et aux libertés des personnes. Il semble être une autre source de discrimination envers les psychiatrisés. Il perpétue les mythes de la violence et des «médicaments miracles». La coercition est une intrusion dans la vie privée; elle nuit au cheminement des individus.

De plus, les OTC changent en quelque sorte la notion de "consentement». Elles ne mènent pas à un consentement libre dans le vrai sens du mot puisqu'elles deviennent monnaie d'échange pour la «liberté». Une fois qu'un individu accepte une OTC, il peut y être lié pendant longtemps.

Enfin, la mise en place des OTC ne règle toujours pas la problématique complexe qu'est le manque de services aux psychiatrisés, que ce soit au niveau de la santé mentale ou de l'amélioration des conditions de vie. Le manque de logement, le faible revenu, le manque d'emploi, l'insuffisance de services de soutien, voilà des questions qui sont toujours en suspens et qui sont pourtant au coeur du problème des psychiatrisés.

La question des OTC s'avère complexe. Cet article a pour but de décrire cette nouvelle loi, son contexte, ainsi que les enjeux qui y sont liés.

\section{Cadre conceptuel}

\section{Historique}

Cette approche d'intervention a fait surface au cours des années 70 aux Etats-Unis, puis elle s'est répandue au cours des années 80. À partir des années 90, elle faisait partie des agendas politiques de tous les États américains (Boudreau et Lambert 1993 : 57-58). C'est ainsi qu'une quarantaine d'États ont en vigueur un type ou 
"Le traitement communautaire obligatoire porte plusieurs noms : community treatment order (CTO), involuntary outpatient treatment, compulsory community treatment, community commital, civil commitment..., traitement externe involontaire, ordonnance de traitement communautaire..." un autre de traitement communautaire obligatoire (CMHA-OD 1998a : 5; Fox Wieselthier s.d. c : 1). La Nouvelle-Zélande est un autre pays qui a adopté ce mode coercitif de traitement (Boyle $1999: 1)$.

La Saskatchewan a été la première province canadienne à imposer une telle loi dès 1995 (CMHA-BC 1997 :1). En 1998, la Colombie-Britannique a suivi avec une loi similaire (Mad Nation s.d. e : 2). Le Manitoba suivra bientôt (No Force Coalition 2000 : 1). Après avoir débattu de la question, le Nouveau-Brunswick a plutôt choisi d'améliorer les services communautaires offerts aux individus et aux familles des psychiatrisés; en même temps, il clarifiait et renforçait l'Acte de santé mentale de la province (Mallen 2000 : 2; No Force Coalition 1999a : 2).

En Ontario, la question a été lancée «publiquement» en 1990 par le sous-comité législatif du comité provincial en santé mentale, et ce, par l'entremise d'un questionnaire sur les services en santé mentale (Boudreau et Lambert 1993 : 58). Richard Patton, un député libéral d'Ottawa Centre, a proposé les OTC au parlement en 1997 : il a présenté les lois 111 (1997) et 78 (1998); elles ont toutes deux été abandonnées après avoir passé la deuxième lecture (Queen's Park 1999). Le parti conservateur de Mike Harris a tôt fait de reprendre l'idée en incluant le traitement obligatoire dans ses promesses électorales (Ontario PC 1999 : 28). C'est une promesse qu'il a bel et bien tenue. Le $1^{\text {er }}$ décembre 2000, la loi 68 était proclamée (OMH 2000d : 1).

\section{Définition}

Le traitement communautaire obligatoire porte plusieurs noms : community treatment order (CTO), involuntary outpatient treatment, compulsory community treatment, community commital, civil commitment (CMHA-NO 1998 :1; CMHA-OD 1998b :1), traitement externe involontaire, ordonnance de traitement communautaire (OTC). Les psychiatrisés, eux, préferent le terme de leash law (Weitz 1999).

L'ordre de traitement communautaire obligatoire est un processus légal et psychosocial qui exige qu'un psychiatrisé vivant dans la communauté suive un traitement. Si l'individu refuse de 
suivre le traitement prescrit — s'il ne prend pas ses médicaments ou s'il ne se présente pas à ses rendez-vous - il risque le retour à l'hôpital ou dans un institut psychiatrique (CMHA-OD 1998b : 1; Boudreau et Lambert 1993 : 57).

En général, un «traitement» se définit par la prise de médicaments (Sheid-Cook 1991 : 50) mais il peut aussi inclure la participation à des programmes de jour, à de la thérapie individuelle ou de groupe, à des programmes vocationnels et éducationnels, à la supervision ainsi qu'à l'utilisation de services d'accompagnement communautaire. Le lieu de résidence, la gestion d'argent, tout comme la question de l'utilisation de drogues ou d'alcool peuvent aussi être des aspects inclus dans l'entente de traitement (Boudreau et Lambert $1993: 58$ ).

Maintenant que nous en savons davantage au sujet des OTC, passons à la description du projet de loi ontarien, la loi 68 .

\section{La loi 68 : loi en souvenir de Brian Smith}

"La nouvelle loi facilite le traitement des psychiatrisés en élargissant les critères d'évaluation et d'internement : la chronicité de la maladie est maintenant un critère et non seulement le danger que présente la personne..."
La loi 68 vient modifier l'Acte en santé mentale (ASM), tout comme la Loi sur le consentement au traitement, pour permettre les ordonnances de traitement communautaire. De plus, des changements de terminologie ont été apportés afin de clarifier certaines expressions ambiguës (OMH 2000c : 1-2).

La nouvelle loi facilite le traitement des psychiatrisés en élargissant les critères d'évaluation et d'internement :la chronicité de la maladie est maintenant un critère et non seulement le danger que présente la personne (OMH 2000b,c : 1; Gouv. Ont. 2000 : 2). Cet ajout à l'article 15 de l'Acte permet aux médecins «de présenter une demande d'évaluation psychiatrique» (Ontario 2000 : 2) si la personne qu'ils ont examinée :

- a reçu des traitements psychiatriques par le passé pour une maladie mentale sévère et persistante qui, lorsqu'elle n'est pas traitée, peut mener la personne à s'infliger des lésions corporelles graves ou à les infliger à d'autres; la maladie peut mener 
à une détérioration physique ou mentale, ou à un affaiblissement physique sérieux;

- a déjà démontré une amélioration de la santé suite au traitement;

- a un historique qui démontre que de telles conséquences se sont déjà produites suite à une détérioration mentale;

- souffre de la même maladie pour laquelle elle s'est faite traiter par le passé;

- est jugée incompétente pour consentir au traitement; un substitut abrogé consent alors à l'évaluation (Ontario 2000 : 2; OMH 2000b : 2).

La nouvelle loi permet aussi à un juge de paix d'ordonner l'évaluation psychiatrique d'une personne s'il a l'impression que l'information soumise correspond aux critères de certification proposés par la loi (Ontario $2000: 3$ ).

\section{Les ordonnances de traitement communautaire}

L'une des modifications principales de l'ASM s'avère l'ajout de mécanismes permettant les OTC. L'ASM identifie les critères d'imposition d'une OTC, le contenu de l'ordonnance, la durée

"Les OTC peuvent être offertes comme une alternative moins restrictive que l'hospitalisation, suite à la recommandation d'un médecin ou d'un psychiatre; ou bien, elles peuvent être offertes comme condition imposée à la sortie du patient de l'hôpital, si ce dernier consent à ce mode de traitement...» et les conditions entourant les OTC, tout comme les obligations de la personne et ses droits. C'est ainsi que nous résumerons les points principaux des OTC.

\section{Dans quelles circonstances les OTC peuvent-elles être prescrites?}

Les OTC peuvent être offertes comme une alternative moins restrictive que l'hospitalisation, suite à la recommandation d'un médecin ou d'un psychiatre; ou bien, elles peuvent être offertes comme condition imposée à la sortie du patient de l'hôpital, si ce dernier consent à ce mode de traitement (Gouv. Ont. 2000 :2; Ontario $2000: 6$ ).

\section{Critères d'admissibilité et d'imposition}

La personne doit avoir été admise dans un établissement psychiatrique à au moins deux occasions différentes, ou avoir été 
hospitalisée pour une durée de 30 jours ou plus durant les trois années précédentes. De plus, il doit y avoir un plan de traitement communautaire en place; la personne (ou son décideur substitut) doit l'avoir accepté. Afin de prescrire une OTC, il faut qu'un médecin ou un psychiatre ait examiné le patient au cours des 72 dernières heures (OMH 2000c : 3; Ontario 2000 : 6).

Le médecin peut émettre une OTC s'il croit que la personne souffre d'une maladie mentale grave et qu'elle a besoin d'un traitement et d'une supervision continus dans la communauté sans quoi elle pourrait s'infliger des lésions corporelles ou en infliger à d'autres ou encore subir une détérioration physique ou mentale sérieuse. De plus, il faut que la personne soit en mesure de se conformer au traitement, que les services appropriés soient disponibles et que la personne (ou son décideur substitut) consente au traitement. Le médecin doit s'assurer que la personne ait consulté un conseiller en matière de droit et qu'elle ait été informée de ses droits (OMH 2000c : 3-4; Ontario 2000 : 6-7).

\section{Obligations du patient sous une OTC}

"Le patient sous une OTC a le devoir de se présenter à ses rendezvous chez les personnes désignées dans le plan de traitement; il doit suivre les conditions imposées dans son plan de traitement..."
Le patient sous une OTC a le devoir de se présenter à ses rendezvous chez les personnes désignées dans le plan de traitement; il doit suivre les conditions imposées dans son plan de traitement (OMH 2000c : 4; Ontario $2000: 7$ ).

S'il ne s'y conforme pas, le médecin peut servir un ordre d'évaluation psychiatrique après avoir fait les efforts nécessaires pour trouver la personne; il doit l'informer (ou informer son décideur substitut) qu'elle n'a pas observé les conditions imposées par l'OTC; il doit l'avertir des conséquences possibles de ses actes; puis il doit l'aider à se conformer aux termes décrits dans l'OTC (OMH 2000c : 4; Ontario $2000: 8$ ).

\section{Durée des OTC et renouvellement}

La première OTC peut être d'une durée de six mois et peut être renouvelée pour des périodes de six mois à la fois, si la personne répond toujours aux critères d'imposition. Une OTC peut être renouvelée jusqu'à un mois suivant l'expiration de l'OTC précédente. Les amendements apportés à la loi permettent à cette 
personne (ou à son décideur abrogé) de se faire évaluer pour voir si l'OTC est encore nécessaire. À chaque fois, l'individu (ou son substitut) peut en appeler d'une décision de renouvellement auprès de la Commission du consentement et de la capacité. Il est aussi possible pour un patient (ou un substitut) de retirer son consentement. Pour ce faire, il doit en avertir le médecin qui évaluera la condition de l'individu et décidera s'il doit être hospitalisé ou s'il peut vivre dans la communauté sans être sujet à une OTC. Si la personne refuse de se faire évaluer, le médecin pourra ordonner un examen psychiatrique (s'il le juge nécessaire) (OMH $2000: 4$; Ontario $2000: 8-9$ ).

\section{Responsabilités du médecin et plan de traitement}

Le médecin est responsable de la supervision du plan de traitement. Puisque ce sont les équipes multi et interdisciplinaires d'intervention en santé mentale qui auront généralement la tâche de s'occuper des gens sous OTC, ce sera sans doute le psychiatre de l'équipe qui coordonnera le tout.

\section{OTC : enjeux pratiques et éthiques}

L'application de cette loi soulève plusieurs questions pratiques et éthiques, particulièrement en lien avec le consentement émis par

« La nouvelle loi proposée par le gouvernement semble bien paradoxale. Même si elle porte le nom d'ordonnance de traitement obligatoire, elle stipule que le patient doit consentir à ce traitement...» les patients. La nouvelle loi proposée par le gouvernement semble bien paradoxale. Même si elle porte le nom d'ordonnance de traitement obligatoire, elle stipule que le patient doit consentir à ce traitement (Ontario 2000 : 6). Puisqu'il en est ainsi, pourquoi appeler cela «traitement obligatoire»? En lisant attentivement la loi, nous remarquons que le patient doit accepter l'OTC et les conditions qui y sont liées ou bien rester à l'hôpital. Le consentement perd donc beaucoup de sa valeur et devient une forme de traitement obligatoire, comme l'indique son nom : il devient une condition pour la sortie de l'hôpital, une condition à la liberté.

Nous pouvons aussi remarquer que même lorsque la personne retire son consentement et refuse de se faire examiner, un examen psychiatrique doit être complété et c'est le médecin qui a le dernier 
mot quant au traitement du patient et qui décide si la personne est en mesure de vivre sans OTC (Ontario 2000 : 6-7).

En plus de présenter certaines contradictions et certains dilemmes éthiques, la loi 68 présente aussi des problèmes pratiques. Comment cette loi sera-t-elle interprétée? De quelle façon serat-elle mise en pratique? Il y a plusieurs «trous» dans cette loi et le gouvernement devra développer des procédures claires afin de pouvoir la mettre en oeuvre. Quelles seront les procédures pour imposer une OTC? Comment sera-t-elle présentée au patient? Quelles seront les conséquences si le client ne se conforme pas au traitement? Voilà, en somme, quelques questions importantes pour lesquelles nous n'avons pas encore de réponses.

Maintenant que nous connaissons un peu mieux la loi, passons aux acteurs impliqués dans le débat et à leurs arguments.

\section{Les acteurs}

Nous diviserons cette partie entre ceux qui sont "pour» et ceux qui sont «contre». Nous présenterons les arguments apportés par les deux groupes.

\section{Les partisans}

Si le concept de traitement communautaire obligatoire a fait autant de chemin au cours des dernières décennies, il faut certainement se tourner vers les "gros joueurs» américains, soit Fuller Torrey, le Treatment Advocacy Center (TAC) et le National Alliance for the Mentally Ill (NAMI) - un groupe de familles des psychiatrisés, groupe qui organise des campagnes à travers les États-Unis afin de convaincre les gens de passer des lois légalisant le traitement coercitif (Mad Nation s.d.d :2). Les compagnies pharmaceutiques qui les subventionnent ont d'ailleurs tout à gagner face à l'implantation des OTC (Fox Wieselthier et Allen 1999:3; Mad Nation s.d.c : 2). En Ontario, les OTC sont promues par le Schizophrenia Society, une branche de NAMI (Boudreau et Lambert 1993 : 70), 
"Quels sont les arguments utilisés pour justifier le traitement involontaire?

Premièrement, il y a le besoin d'équilibrer les droits $d u$ patient avec ceux de la société, laquelle a droit à la sécurité... Le droit au traitement en est un autre..." par le gouvernement provincial, par divers politiciens (Ontario, 1998: 1-4), ainsi que par divers professionnels de la santé, dont le Ontario Medical Association (CMHA-OD 1998a : 6).

Quels sont les arguments utilisés pour justifier le traitement involontaire? Premièrement, il y a le besoin d'équilibrer les droits du patient avec ceux de la société, laquelle a droit à la sécurité (CMHA-OD 1998a : 6; Mallen 2000 : 1). Le droit au traitement en est un autre: il stipule que la personne a droit à la santé ainsi qu'à une qualité de vie décente (CMHA-OD 1998 : 6,8; CMHANO 1998 :2). Les OTC offriraient une alternative moins restrictive aux psychiatrisés en réduisant les chances que la santé des personnes se détériore au point qu'elles soient obligées de se rendre à l'hôpital, endroit où les droits sont encore moins respectés (CMHA-NO $1998: 2-3)$.

D'autres avantages seraient liés aux OTC. Elles permettraient de garder les gens hors des hôpitaux, de réduire le phénomène de la porte tournante, de diminuer le taux de réhospitalisation et de crises suite à l'abandon du traitement. De plus, l'argent épargné par la réduction du taux d'hospitalisation pourrait être utilisé afin d'améliorer les services communautaires (CMHA-NO 1998 : 2-3,5).

Finalement, la façon d'utiliser les médicaments est importante. Lorsqu'ils sont pris de façon consistante, ils permettent aux professionnels d'être plus efficaces dans leur rôle d'intervenants (CMHA-NO 1998).

\section{Les opposants}

Les opposants aux OTC sont nombreux. Plusieurs professionnels de la santé, planificateurs de services et même diverses institutions — dont les hôpitaux — sont contre les OTC. Un grand nombre d'organismes communautaires, dont l'Association canadienne pour la santé mentale et le Mental Health Legal Committee, dénoncent aussi ce projet de loi (CMHA-OD 1998a :7,13). Les groupes de psychiatrisés, dont le Queen Street Patient Council, le Psychiatric Survivor Action Association of Ontario, le No Force Coalition, le Patients Against Coercive Treatment (PACT) s'opposent fortement aux OTC. 
"Pour les opposants, le traitement obligatoire soulève plusieurs enjeux,... le droit à l'égalité, le droit à la sécurité, et le droit à la protection contre une détention arbitraire ou contre un traitement cruel ou injuste."
Il est intéressant de noter que même s'il y a dissension entre les psychiatrisés au niveau des diagnostics, du traitement ou des causes de la maladie mentale, il n'en est pas de même quant à la question du traitement obligatoire. Les OTC ont eu l'effet de créer une union très forte dans cette communauté (Mad Nation s.d.a). Il faut noter que les groupes de psychiatrisés jouent un rôle important dans les politiques en santé mentale et dans l'organisation des services offerts (Blais et al. 1998 : 19). Il semble d'ailleurs que ce soit une campagne orchestrée par des psychiatrisés qui ait mené au rejet des lois 78 et 111 (versions antérieures des OTC) pourtant acceptées en deuxième lecture (Weitz 2000).

Pour les opposants, le traitement obligatoire soulève plusieurs enjeux, liés, entre autres, aux droits et libertés : le droit à l'égalité, le droit à la sécurité, et le droit à la protection contre une détention arbitraire ou contre un traitement cruel ou injuste. Le traitement obligatoire serait une autre source de discrimination face aux psychiatrisés. Il perpétue la croyance que ces personnes sont violentes, sans prévention et incapables de prendre soin d'elles-mêmes. La coercition constitue une intrusion dans la vie privée; elle a des effets néfastes sur les individus et sur leur cheminement personnel.

Examinons donc les arguments des opposants de plus près. Ces derniers dénoncent deux mythes populaires soutenus par les partisans des OTC, soit celui de la violence et celui des «médicaments miracles».

«Dans les médias, près de la moitié (46\%) des articles touchant la maladie mentale sont reliés à des crimes» (No Force Coalition 1999b : 1). Il n'est donc pas surprenant que quatre Canadiens sur cinq $(80 \%)$ croient que les personnes éprouvant des troubles de santé mentale sont dangereuses et que le potentiel de leur violence soit l'argument principal utilisé pour la promotion et pour la défense des OTC. En présentant son projet de loi 78 et pour en faire la promotion, Richard Patten a utilisé, notamment, les exemples de Brian Smith et d'EdmondYu (schizophrène tué par un policier à Toronto) (Ontario $1998: 2-3$ ). Fuller Torrey et ses collègues utilisent la même stratégie pour vendre le traitement obligatoire (Fox Wieselthier s.d. a : 1). 
Pourtant, l'ACSM et le Clarke Institute of Psychiatry (No Force Coalition 1999b :2) rapportent que seul un crime sur vingt-cinq (4\%), y compris les crimes violents, est commis par des individus ayant un diagnostic psychiatrique. Puisqu'il est estimé qu'un Canadien sur cinq souffre de trouble de santé mentale, ce taux est donc très minime (No Force Coalition 1999b : 2).

Certaines recherches stipulent qu'il y a un plus haut taux d'arrestation et d'incarcération chez les psychiatrisés. Cependant, l'association entre le crime et les maladies mentales sérieuses disparait lorsque les facteurs d'âge, de sexe, de diagnostics de personnalité anti-sociale, de désordres de comportement (enfants) et de toxicomanie sont contrôlés. L'abus de substances (diagnostic multiple tels la maladie mentale et l'alcoolisme) ainsi qu'une personnalité anti-sociale semblent les facteurs les plus souvent associés au crime (Hiday $2000: 3$ ).

Somme toute, le danger que présentent les psychiatrisés semble grandement exagéré. Dans sa revue de littérature, Monahan (1992) souligne qu'au moins $90 \%$ des psychiatrisés ne sont pas violents (CMHA-OD 2000a : 5).

\section{Les «médicaments miracles»}

"La prise de médicaments s'avère cruciale... Les effets secondaires des médicaments sont dévastateurs, déplaisants et parfois même permanents..."
La prise de médicaments s'avère cruciale dans la question du traitement obligatoire (CMHA-BC 1997 : 3). Nous estimons qu'entre 20 et $40 \%$ des psychiatrisés vivant en communauté ne prennent pas leurs médicaments. Les opposants estiment que cela fait suite à la négation ainsi qu'à un manque de compréhension face à la maladie. Cependant, il existe plusieurs autres raisons (CMHA-BC 1997 : 5). Les effets secondaires des médicaments sont dévastateurs, déplaisants et parfois même permanents : les tremblements, la dyskénisie tardive, la bouche sèche, les dysfonctions sexuelles, la fatigue, la perte d'intérêt, la diminution des réponses émotionnelles, le retrait émotionnel et l'indifférence affective n'en sont que quelques-uns (QSPC s.d. : 3; McCubbin 2000 : 3). Entre 62 et $90 \%$ des individus qui prennent des neuroleptiques éprouvent des malaises causés par les médicaments (McCubbin $2000: 3$ ). 
"De plus, l'efficacité des drogues prescrites s'avère très discutable. "
De plus, l'efficacité des drogues prescrites s'avère très discutable. Très peu de données démontrent clairement l'efficacité des médicaments. L'une des études les plus positives sur les neuroleptiques estime que de 30 à $50 \%$ des patients ont une rechute en utilisant des drogues, comparativement à $70 \%$ pour ceux qui n'en prennent pas (Breggin 1991 : 63). Par ailleurs, une revue de 16 études contrôlées portant sur les nouveaux antidépresseurs démontre que leur taux d'efficacité est encore plus bas : il n'y avait, dans $62 \%$ des cas, aucune différence entre les individus qui prenaient les vrais médicament et ceux qui consommaient les placebos (Breggin 1991 : 158-159).

Quoique la négligence de la prise de médicaments ait son influence sur les rechutes, des études récentes ont démontré que ce n'est pas la cause première des réhospitalisations; des facteurs sociaux sont souvent à l'origine des crises. Les problèmes relationnels, les problèmes de logement et l'isolement social expliquent $39 \%$ des cas, tandis que $31 \%$ des cas sont d'origine médicale (dont la négligence des médicaments). Cela suggère donc qu'il faudrait améliorer le soutien social offert à cette population, au lieu de la médicamenter (CMHA-BC 1997 : 3).

\section{Législation actuelle}

Les opposants soulèvent d'autres arguments. Ils soutiennent que nous possédons déjà la législation nécessaire pour remplir les objectifs des OTC. En effet, la Loi sur la santé mentale donne les pouvoirs nécessaires à l'État et aux médecins pour assurer la protection de la société et de l'individu si cette personne semble dangereuse pour les autres ou pour elle-même. Elle permet de détenir une personne qui présente des comportements «dangereux», de la restreindre et de lui imposer une évaluation psychiatrique. Tout cela peut se faire contre le gré de l'individu (Hoffman 1997 : 171-175).

De plus, la Loi sur le consentement au traitement permet de traiter les individus sans leur consentement dans des situations d'urgence. Elle permet aussi de désigner un décideur substitut pour prendre 
des décisions de traitement si la personne est jugée «incapable» de ce faire (Hoffman $1997:$ 2).

La Loi sur la prise de décisions au nom d'autrui permet «de préciser et d'étendre les droits des adultes mentalement incapables de prendre des décisions» (MPG 1994 :6). Utilisée convenablement, cette loi éviterait bien des conflits quand vient le temps de traiter une personne «incapable». Lorsqu'elle est "capable», une personne peut choisir d'avance qui sera son décideur substitut, lequel aura le pouvoir de consentir ou de refuser un traitement à son nom. Elle peut aussi choisir les traitements à recevoir si elle devenait «incapable», par exemple, l'évaluation psychiatrique, l'utilisation de médicaments, etc. (MPG 1994 : 39-41). Ses voeux doivent être respectés en cas de «maladie» et d'incapacité.

En plus de toutes les lois déjà mentionnées qui permettent de garder un équilibre entre la sécurité de la société et de l'individu et d'identifier les désirs de ce dernier, la Loi sur la santé mentale contient une clause équivalente au traitement obligatoire involontaire, soit «l'absence autorisée». Cette permission de s'absenter de l'hôpital peut être révoquée si la personne répond aux critères de certification (évaluation psychiatrique de 72 heures) ou si le médecin juge que diverses conditions assorties à cette absence n'ont pas été suivies (LSM 1990, art. 27; Schneider 1996 : 110-111). Ce type d'absence permet à l'hôpital d'exercer un certain contrôle sur le client, tandis que ce dernier peut se libérer quelque peu des contraintes de l'institution (Bartlett 1986).

\section{La Charte canadienne des droits et libertés}

Lorsque nous discutons des aspects légaux des OTC, il faut tenir compte d'un autre document : la Charte canadienne des droits et libertés. En effet, plusieurs opposants estiment que le traitement involontaire s'oppose à diverses articles de la Charte. Les points soulevés concernent le droit à l'égalité des droits, la détention arbitraire, le traitement cruel et injuste, et la discrimination. 


\section{La psychiatrie : une science arbitraire?}

"En ce moment, les psychiatrisés sont les seules personnes au Canada qui peuvent être détenues sans avoir commis de crimes ou d'actes dangereux..."
L'article 9 de la Charte souligne qu'une personne ne peut pas être retenue ou emprisonnée de façon arbitraire (CCDL 1982; Schneider 1996 : 451). En ce moment, les psychiatrisés sont les seules personnes au Canada qui peuvent être détenues sans avoir commis de crimes ou d'actes dangereux (CMHA-OD 1998b : 7; QSPC s.d.:4). Pourtant, les psychiatres ne peuvent pas déterminer avec justesse le danger que représentent les individus. Les recherches ont démontré que les professionnels de la santé ont tort dans $80 \%$ de leurs prédictions (CMHA-OD 1998a : 7).

Le diagnostic même de la maladie mentale est ambigu. La définition, la fiabilité et l'utilité de termes tels que maladie mentale, schizophrénie et normalité sont conflictuelles. Ce qui est normal dans une culture peut être inacceptable dans une autre ou encore, changer d'une époque à une autre (Rosenhan 1973 : 250). Les diagnostics psychiatriques sont imprégnés de jugements et de valeurs (Borovoy 1988 : 183).

Plusieurs études ont relevé un taux de dissension élevé entre les psychiatres quand vient le temps d'imposer un diagnostic. Selon Rosenhan (1973), les psychiatres n'ont pas été en mesure de différencier les pseudo-patients des «vrais» psychiatrisés. D'autres études démontrent que les psychiatres ont tendance à imposer des diagnostics plus sévères aux individus qui expriment des visions politiques plus radicales et qui critiquent davantage le système médical (Borovoy 1988 : 187).

En plus de détenir le pouvoir d'évaluer le danger que représentent les individus et de poser des diagnostics psychiatriques, les professionnels de la santé ont le pouvoir de décider du degré de compétence détenu par le patient, dont sa capacité de consentir au traitement. Guidés par leur désir de "guérir» des maladies, les intervenants sont susceptibles d'interpréter le refus de traitement comme preuve d'incapacité (Borovoy 1988 : 198).

\section{Égalité des droits}

L'article 15 de la Charte explique que nous sommes tous égaux devant la loi. Nous avons tous le droit d'être protégés par la loi et 
d'en tirer profit de façon équitable, et ce, sans discrimination basée sur la race, le sexe, la nationalité, la culture, l'âge, la maladie mentale ou physique (CCDL 1982 : art. 15; Schneider 1996 : 452). C'est donc dire que toute personne a le droit d'accepter ou de refuser un traitement médical (CMHA-OD 1998a: 4). Cependant, l'article 15(2) souligne le bien-fondé des lois et des activités qui ont pour but l'amélioration des conditions de vie des individus désavantagés à cause de leur race, de leur sexe, de leur religion ou de leur «incapacité» mentale ou physique (CCDL 1982 : art. 2; Schneider $1996: 452-453$ ).

Dans cette optique, la loi des OTC serait conforme à la Charte, tout comme les lois sur le consentement au nom d'autrui. La maladie mentale semble justifier l'intrusion à la liberté personnelle des individus. En tenant compte du fait que la psychiatrie est une science inexacte, en connaissant les dangers reliés au traitement et en voyant les effets de la coercition, est-ce vraiment justifiable? Les effets semblent d'ailleurs contrevenir à l'article 7 de la Charte, soit au droit à la liberté et à la sécurité personnelle.

Le traitement obligatoire enfreint le droit à ne pas être détenu arbitrairement, le droit à l'égalité, le droit à la liberté et le droit à la sécurité. À cette liste, s'ajoute le droit à la liberté de croyance et de pensée.

L'article 2 de la Charte soutient que nous avons tous droit à la liberté de parole, de pensée, d'opinion, de croyance, de conscience et de religion (CCDL 1982 : art. 2; Schneider 1996 : 449). Seule la façon de manifester ses pensées limite ce droit. Puisque c'est la nature même des pensées et des croyances des schizophrènes qui caractérise le plus leur maladie, cet article de la Charte s'avère très pertinente (Godsen 1997 :6). Dans une société démocratique, ne devrions-nous pas tolérer et même encourager la diversité (Borovoy 1988 : 184)? Ne devrions-nous pas protéger les droits de ces individus? N'est-ce pas aller à l'encontre de ces droits fondamentaux que d'imposer un traitement à une personne qui ne contrevient pas à la loi, mais qui exprime des valeurs et des pensées bizarres, «superflues», «irrationnelles», etc. (Borovoy 1988 : 184)? L'individu devrait être libre de penser et de croire ce qu'il 
veut bien, et ce, sans interférence. Il n'y a pas d'intérêt collectif qui justifie une telle intrusion.

Fondamentalement, le patient a droit à la dignité, à l'intégrité et à l'autonomie. Le traitement forcé s'avère peu justifiable; une pratique éthique, responsable et rationnelle ne devrait pas imposer un traitement, mais plutôt offrir du soutien à la personne (McCubbin $2000: 5$ ).

Maintenant que nous avons soulevé divers thèmes et enjeux liés aux OTC, nous discuterons des implications au niveau de l'intervention.

\section{Coercition et implications au niveau de l'intervention}

"La coercition est le mode de traitement privilégié par le traitement obligatoire. "

"Cela nous mène à la question du manque de ressources et de services adéquats." "

\section{Efficacité}

La coercition est le mode de traitement privilégié par le traitement obligatoire. Mais que peut-on dire de ce choix d'intervention? Très peu d'études ont été faites sur le traitement obligatoire. En général, lorsque nous comparons les clients participant au traitement obligatoire à ceux recevant les mêmes services, mais de façon volontaire, nous ne notons aucune différence, que ce soit au niveau de la conformité, de la continuité du traitement, ou au niveau des taux d'hospitalisation. Les taux d'arrestation et les taux d'actes violents sont pareils, également (Bazelon Center 2000 :2). Ce qui ressort de ces recherches, c'est que le traitement forcé n'a aucune valeur intrinsèque. Là où il y a eu amélioration, c'est au niveau de la disponibilité des services. Ces ordonnances ont forcé le système de santé mentale à s'impliquer davantage auprès des psychiatrisés afin de leur offrir des services acceptables et nécessaires pour leur bien-être. Cela expliquerait donc pourquoi diverses études ont obtenu des résultats positifs face au traitement obligatoire (Bazelon Center $2000: 3$ ).

\section{Manque de ressources et de services adéquats}

Cela nous mène à la question du manque de ressources et de services adéquats. Pour mettre en place les OTC, une gamme de 
"Il $y$ a divers coûts associés au traitement forcé et au non-respect du choix ou du désir de la personne." ressources qui répondent à l'ensemble des besoins des psychiatrisés doivent être disponibles (CMHA-ON 2000b : 12). Pourtant, il semble peu probable que ce sera le cas. Déjà, nous n'avons pas assez de services pour répondre aux besoins des clients volontaires. Par exemple, les gens qui veulent recevoir des services d'accompagnement communautaire sont sur des listes d'attente de trois à cinq ans (Smith 2000). Plusieurs personnes vont chercher de l'aide à l'hôpital ou ailleurs, mais se la font refuser. Cela a été le cas de Yu avant qu'il ne se fasse tirer (CMHA-BC 1997 : 5). Ainsi, dans une étude effectuée en Colombie-Britannique, $42 \%$ des répondants disent s'être fait refuser des services; de ce nombre, $43 \%$ ont dû se faire certifier par après (CMHA-BC 1997 : 5). Par ailleurs, le manque de lits fait en sorte que pour recevoir des services, les gens doivent être en crise; il y a donc peu de prévention. L'aménagement de ressources pour la création de services obligatoires diminuera davantage les services disponibles pour les clients volontaires (Fox Wieselthier et Allen 1999 : 2)

\section{Effets sur la relation thérapeutique et nouveau rôle des intervenants}

Il y a divers coûts associés au traitement forcé et au non-respect du choix ou du désir de la personne. Le traitement médical engendre la passivité et la résignation chronique (McCubbin $2000: 4)$. En effet, l'imposition d'un traitement par un professionnel, particulièrement l'utilisation des médicaments, a tendance à renforcer l'autorité du médecin, puis le rôle du «malade» chez la personne. Cette dernière souffre d'une «maladie» et le médecin a le «remède». Elle apprend à se soumettre; elle perd son pouvoir; elle devient impuissante (Breggin 1991 :59). La coercition enlève le choix à la personne d'apprendre à connaitre sa maladie et de trouver des moyens efficaces pour la maitriser par elle-même (CMHA-BC 1997 : 6; Lunt, s.d. : 2). La liberté et le sentiment d'avoir le contrôle sur sa propre vie sont essentiels pour une vie satisfaisante et donc pour le rétablissement (Lunt s.d. : 2).

Que la personne soit compétente ou non, la coercition l'infantilise et l'humilie. Elle crée de la colère, de la résistance au traitement, puis elle nuit à la relation thérapeutique, qui est 
pourtant essentielle au rétablissement des gens (McCubbin 2000 : 4; CMHA-BC 1997 : 4). En effet, la coercition introduit un élément d'antagonisme dans la relation et crée une nouvelle distance entre l'intervenant et le client; cette nouvelle «arme» détenue par l'intervenant se traduit par un plus haut taux de méfiance de la part du client, ce qui peut avoir un effet négatif sur son désir de discuter de ses symptômes, des effets des médicaments ou de sa satisfaction face à ces services. La confiance et l'alliance thérapeutique en souffrent (PRA $1998:$ VI : d).

C'est l'une des raisons pour laquelle plusieurs intervenants sont réticents face à une telle loi; ils ne l'appliqueraient que s'ils y étaient obligés. La plupart des intervenants disent travailler «avec la personne» et valorisent la relation d'aide. Ils croient que la coercition s'avère très néfaste pour l'alliance thérapeutique. Ils ne veulent pas jouer le rôle de policier ou d'agent de probation (CMHA-NO 1998 : 5). En effet, les OTC créeraient un système de probation en santé mentale; les intervenants seraient alors appelés à surveiller les patients et à rapporter leurs "écarts» de conduite aux autorités nécessaires (CMHA-OD 1998b : 8) : de quoi nuire à la relation d'aide, évidemment.

De plus, la coercition éloigne et décourage les gens à utiliser les services volontaires lorsqu'ils en ont besoin de peur d'être soumis à un traitement obligatoire. Éviter les services de santé peut mener à la détérioration de leur état de santé, jusqu'au point où ils devront recevoir des traitements forcés.

"Somme toute, la force, c'est de la violence. »
Somme toute, la force, c'est de la violence. Elle encourage l'impuissance. Elle enlève l'estime de soi et chasse tout espoir de rétablissement. Elle nuit au bien-être. (Mad Nation s.d. b : 3). Comme le ditWieselthier (s.d.b) : If it isn't voluntary, it isn't treatment!

\section{Conclusion}

Face aux OTC, que pouvons-nous faire en tant qu'intervenants? D'une part, si nous travaillons avec des individus assujettis à une 
"...nous devrions tenter de leur offrir le maximum de contrôle et de choix dans leur traitement.»
OTC, nous devrions tenter de leur offrir le maximum de contrôle et de choix dans leur traitement. D'autre part, puisque la loi demande que tous les partis soient d'accord face au plan de traitement d'une personne, nous avons la possibilité de suggérer des alternatives, de montrer notre désaccord et même de refuser de traiter une personne sous une OTC.La promotion de méthodes alternatives aux OTC serait de mise.

Selon les opposants aux OTC, nous devrions encourager le développement de lois et de services afin d'améliorer les conditions de vie des psychiatrisés et de mieux répondre à leurs besoins. Nous devrions attaquer les problèmes réels auxquels les psychiatrisés font face, soit la stigmatisation, le manque de logement abordable, le faible revenu qui leur est accordé, le peu d'emplois qui leur sont disponibles, tout comme la pénurie de programmes et de services en santé mentale (QSPC s.d. : 2; No Force Coalition 2000 : 8).

C'est ainsi que nous devrions offrir une gamme de services et de ressources communautaires qui répondent aux besoins des psychiatrisés. Ces ressources devraient promouvoir le respect, la dignité, l'auto-détermination et l'empowerment du client, tout en étant orientées vers la réhabilitation et le rétablissement (Mad Nation s.d. b : 3; Copeland 1999-2000 : 78; CMHA-OD 1998a : 7).

De plus, nous devrions réviser notre système d'assistance sociale et offrir plus de programmes de formation et d'emplois. Nous devrions aussi encourager la création d'entreprises auto-gérées par les psychiatrisés (No Force Coalition 2000 : 8).

La sensibilisation du public face à la maladie mentale ainsi que l'éducation des psychiatres et des intervenants quant aux lois en santé mentale seraient de mise, puisque plusieurs d'entre eux ne semblent pas être au courant du pouvoir législatif qu'ils détiennent (CMHA-OD 1998a : 7; No Force Coalition 2000 :9; Copeland 1999-2000 : 7). Bref, l'amélioration des services et des conditions de vie des psychiatrisés, tout comme la sensibilisation des professionnels et du public seraient des alternatives définitivement plus humaines que l'imposition des OTC... et sans doute plus efficaces. 


\section{Bibliographie}

ASSOCIATION CANADIENNE POUR LA SANTÉ MENTALE (ACSM, 1995), 42e rapport annuel, OTTAWA,ACSM.

BARTLETT, Peter (1986), Mental Health Law. The Advocate's Manual, Toronto, Community Legal Education Ontario.

BAZELON CENTER FOR MENTAL HEALTH LAW (2000), Studies of Outpatient Commitment Are Misused. The Effects of Outpatient Commitment on Use of Mental Health Services Are Greatly Exaggerated, http://www.bazelon.org/opcstud.html

BLAIS, L., L. MULLIGAN-ROY et C. CAMIRAND (1998), «Un chien dans un jeu de quilles : le mouvement des psychiatrisés et la politique de santé mentale communautaire en Ontario», Revue canadienne des politiques sociales, 42, 15-35.

BOROVOY, Alan (1988), When Freedoms Collide, Toronto, Lester \& Orpen Dennys, 182-200.

BOUDREAU, Françoise et Philip LAMBERT (1993), «Compulsary Community Treatment? I. Ontario Stakeholder's Responses to "Helping Those Who Can't Help Themselves"”, Canadian Journal of Community Mental Health, 12(1), 57-78.

BREGGIN, Peter (1991), Toxic Psychiatry. Why Therapy, Empathy, and Love Must Replace the Drugs, Electroshock, and Biochemical Theories of the "New Psychiary", New York, St. Martin's Press, 464 p.

CANADIAN MENTAL HEALTH ASSOCIATION - BRITISH COLUMBIA DIVISION (CMHA-BC, 1997), Community Committal: Another View, CMHA-BC division, $10 \mathrm{p}$.

CANADIAN MENTAL HEALTH ASSOCIATION - NATIONAL OFFICE (CMHA-NO, 1998), Community Committal: A discussion Paper, CMHA-NO, 11 p.

CANADIAN MENTAL HEALTH ASSOCIATION - ONTARIO DIVISION (CMHA-OD, 2000a), Violence and Mental Illness: A Survey of Recent Literature, Toronto, CMHA, 17 p..

CANADIAN MENTAL HEALTH ASSOCIATION - ONTARIO DIVISION (CMHA-OD, 2000b), Survey of Community Treatment Order Literature, Toronto, CMHA-OD, 18 p.

CANADIAN MENTAL HEALTH ASSOCIATION — ONTARIO DIVISION (CMHA-OD, 1998a), «Community Treatment Orders», Network. Is Patient Freedom Being Violated, 14(2), 313.

CANADIAN MENTAL HEALTH ASSOCIATION - ONTARIO DIVISION (CMHA-OD, 1998b), Position Statement Regarding The Use of Community Treatment Orders for Persons With Mental Illness, Toronto, CMHA-OD, 13 p.

CHARTE CANADIENNE DES DROITS ET LIBERTÉS (CCDL, 1982), http:// canada.justice.gc.ca/Loireg/charte/const_fr.html

COPELAND, R. (1999-2000), Vermont's Vision of a Public System for Developmental and Mental Health Services Without Coercion, Waterbury,Vermont Department of Developmental and Mental Health Services, $13 \mathrm{p}$.

FOX WIESELTHIER, V. (1999), "Connecting the Dots: The Corruption of the Family Mental Health Movement in America», Mad Nation, http://www.madnation.org/vicky/connecting.htm FOX WIESELTHIER, V. (s.d. a), «Five Lies and Distortions E. Fuller Torrey and the Treatment Advocacy Center Use to Sell Force and Coercion», Mad Nation, http://www.madnation.org/ vicky/taclies.htm

FOX WIESELTHIER, V. (s.d. b), «If it Isn't Voluntary it Isn't Treatment», Mad Nation, http:// www.madnation.org/NOFORCE.htm 
FOX WIESELTHIER,V. (s.d.c), «The Simple Truth: Selling Coercion to Ann Landers», Mad Nation, http://www.madnation.org/simpletruth.htm

FOX WIESELTHIER, V. et M. ALLEN (1999), «Forced Treatment Doesn't Work: The Promise of Community Mental Health Service Was Neither Funded Nor Fulfilled», The Washington Post, Aug. 5, A23, dans Mad Nation, http://www.madnation.org/post.htm

GAGNON, J. (2000), Le traitement communautaire obligatoire: une pratique justifiable?, Mémoire déposé à l'École de service social en vue de l'obtention de la maitrise en service social, sous la dir. de Louise BLAIS, Ottawa, Université d'Ottawa. $80 \mathrm{p}$.

GODSEN, R. (1997), «Shrinking the Freedom of Thought: How Involuntary Psychiatric Treatment Violates Basic Human Rights», Journal of Human Rights and Technology, vol. 1, http://www.hri.ca/ doccentre/psychiatric/gos.d.en.shtml

GOUVERNEMENT DE L'ONTARIO (2000), «L'Ontario dépose la loi de Brian pour l'amélioration des traitements de santé mentale et la sécurité des collectivités», \Gouvernement de l'Ontario. Communiqués de presse, Gouvernement de l'Ontario, http://www.newswire.ca/ gouvernement/ontario/french/releases/April2000/25/c6779.html

HIDAY, V. (2000), «Criminalization of the Mentally Ill», Mad Nation, http://www.madnation.org/ research/hiday.htm

HOFFMAN, Brian (1997), The Law of Consent to Treatment in Ontario, Toronto, Butterworth, 238p.

LOI SUR LA SANTÉ MENTALE (LSM), Ontario 1990, chap. M.7.

LUNT, Allen (s.d.), «Outpatient Commitment», Mad Nation, http://www.madnation.org/essays/ lunt.htm

MAD NATION, (s.d. a), «Talking Points», Mad Nation, http://www.madnation.org/news/IOC/ talking.htm

MAD NATION, (s.d.b), «Replacing Outpatient Commitment Initiatives With Strategies That Work to Engage People in Need», Mad Nation, http://www.madnation.org/news/kendra/ strategiesthatwork.htm

MAD NATION, (s.d.c), «Stop Coercion and PACT/ACT Perversion Now. A Mad Nation Essay/ Action Alert», Mad Nation, http://www.madnation.org/text/pact.htm

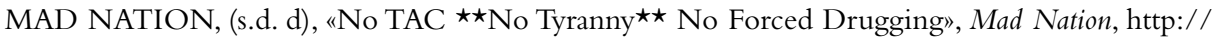
www.madnation.org/news/oppressors/tacfact.htm

MAD NATION, (s.d. e), «British Columbia’s Bill 22», Mad Nation, http://madnation.org/bill22.htm

MALLEN, Caroline (2000), "Ontario to Introduce Stronger Mental Health Law in Spring to Help Patients and Protect Communities», Toronto Star, March 23, dans «CTO Update», QSPC, http:/ /www.icomm.ca/patientsco/update.htm

McCUBBIN, Micheal (2000), Analysis of the Scientific Grounds For Forced Treatment, http:// www.mailbase.ac.uk/lists/radical-psychology-network/2000-01/0050.html

MINISTÈRE DU PROCUREUR GÉNÉRAL (MPG,1994), Guide de la Loi sur la prise de décisions au nom d'autrui, Toronto, Imprimeur de la Reine pour l'Ontario, $51 \mathrm{p}$.

NO FORCE COALITION (2000), «Protest», The No Force Coalition, http://www.icomm.ca/ patientsco/protest.htm

NO FORCE COALITION (1999a), «Dear Member of Parliament of Provincial Government (Ontario)", Dec. 14, http://www.icomm.ca/patientsco/mppcto.htm 
NO FORCE COALITION (1999b), «TheViolent Mental Patient Myth», http://www.icomm.ca/ patientsco/myth1

ONTARIO (2000), Projet de loi 68. Loi à la mémoire de Brian Smith modifiant la Loi sur la Santé mentale et la loi de 1996 sur le consentement aux soins de santé, 21 p. http://www.ontla.on.ca/Documents/ StatusofLegOUT/b068_f.htm

ONTARIO (1998), «Private Member's Public Business. Mental Health Amendment Act», 1998, Ontario Hansard, November 26, $2^{\text {nd }}$ session, 36th Parliment, http://gateway.ontla.co.ca/hansard/ 36_parl/session2/L060A_1.htm

ONTARIO MINISTRY OF HEALTH (OMH, 2000a), Press Release From the Ministry of Health: Ontario to Introduce Stronger Mental Health Law This Spring to Help Patients and Protect Communities, $\mathrm{OMH}, 25$ avril, http://www.newswire.ca/government/ontario/english/releases/March2000/ 22/c6202.html

ONTARIO MINISTRY OF HEALTH (OMH, 2000b), The Next Step: Strengthening Ontario's Mental Health System. Consultation on Proposed Legislative Changes to the Mental Health Act and the Health Care Consent Act, OMH, March 22, http://www.gov.on.ca/health/english/pub/mental/ consultation.html

ONTARIO MINISTRY OF HEALTH (OMH, 2000c), Brian's Law (Mental Health Legislative Reform) 2000, OMH, http://www.gov.on.ca/health/english/pub/mental/brianslaw.html

ONTARIO MINISTRY OF HEALTH (OMH, 2000d), La loi Brian prend effet, OMH, 30 nov., http://www.newswire.ca/government/ontario/french/releases/November2000/30/c9671.html

ONTARIO PC (1999), Plan d'action. Le programme de Mike Harris pour maintenir l'Ontario dans la bonne voie, Toronto, Ontario PC, $55 \mathrm{p}$.

QUEEN'S PARK (1999), «Bill to Help the Seriously Mentally Ill, Retabled», Liberal News Release, May 4.

QUEEN STREET PATIENT COUNSEL (QSPC, s.d.), «Statement of Opposition to “Community Treatment Orders" and Expanded Criteria for Involuntary Admissions to Psychiatric Facilities", CTO Update, http://www.icomm.ca/patientsco/ctonot.htm

ROSENHAN, D.L. (1973). «On Being Sane in Insane Places», Science, 179, 250-258.

SCHNEIDER, Richard (1996), Ontario Mental Health Statutes, Toronto, Carswell, 663 p.

SHEID-COOK, Teresa (1991), "Outpatient Commitment as Both Social Control and Least Restrictive Alternative», The Sociological Quarterly, 32(1), 43-60.

SMITH, M. (2000), Case Manager Training, Ottawa, CMHA-MHCSS

WEITZ, D. (2000), «Who's Really Dangerous? Media Bias — Forced Drugging — Outpatient Committal», Mad Nation, http://www.madnation.org/canada/weitz.htm

WEITZ, D. (1999), «Outpatient Committal/Forced Drugging — A Fashist Threat?», The Outreach Connexion, Jan. 25, dans CTO Update, http://www.icomm.ca/patientsco/cto.htm 